\title{
EDITORIAL
}

Epidemiology and Population Health

\section{Diabetes and blood pressure mediate the effect of obesity on cardiovascular disease}

\author{
Jordi Merino $\mathbb{D}^{1,2,3}$
}

Received: 12 March 2021 / Revised: 26 March 2021 / Accepted: 23 April 2021 / Published online: 17 May 2021

(c) The Author(s), under exclusive licence to Springer Nature Limited 2021

As a major contribution on type 2 diabetes, cardiovascular disease, cancer, and overall mortality, action on obesity is a clinical and public health priority. On the occasion of World Obesity Day, observed annually on March 4, and with the theme of "Every body Needs Everybody", calls on the global and scientific community were made to come together to respect, care, protect, and drive policy change to prevent and improve the life of people living with obesity. Raising awareness of obesity as a global health issue and improve understanding of the root causes, complexities, and consequences of the disease is expected to help inform and design new or more efficient preventive and therapeutic strategies. The identification of causal mediators through which obesity impacts on other metabolic diseases such as cardiovascular disease can offer clinically actionable evidence to minimize the burden of the disease. The paper by Gill et al. [1] in this issue of the journal is a good case in point.

This team of researchers used large-scale genetic association data within a statistical method called multivariable Mendelian randomization to investigate the extent to which systolic blood pressure, diabetes, blood lipids (LDLc, HDLc, and triglycerides), and smoking mediated an effect of body mass index and waist-hip-ratio on risk of coronary artery disease, peripheral artery disease, and stroke. By using multivariable Mendelian randomization, an approach that leverages genetic variants associated with several measured risk factors to simultaneously estimate the causal effect of each of the risk factors on the outcome [2], authors

Jordi Merino

jmerino@mgh.harvard.edu

1 Diabetes Unit and Center for Genomic Medicine, Massachusetts General Hospital, Boston, MA, USA

2 Programs in Metabolism and Medical \& Population Genetics, Broad Institute of MIT and Harvard, Cambridge, MA, USA

3 Department of Medicine, Harvard Medical School, Boston, MA, USA showed that the increased coronary artery disease risk conferred by obesity attenuated after adjusting for intermediate cardiovascular risk factors. The proportion mediated by specific intermediate risk factors was $41 \%(95 \% 18$ to $63 \%$ ) for diabetes, $27 \%$ (95\% CI 3 to $50 \%$ ) for systolic blood pressure, $6 \%$ (95\% CI -20 to $32 \%$ ) for smoking, and $3 \%$ ( -23 to $29 \%$ ) for lipids. When all the mediators were included together in the same model, authors found that the effect of obesity on coronary artery disease attenuated to 1.14 (95\% CI 1.04 to 1.26), and that up to $66 \%$ (95\% CI 42 to 91) of the effect was mediated through these intermediate cardiovascular risk factors. A similar pattern was observed when peripheral artery disease or stroke were used as the outcome, and when waist-to-hip-ratio was the exposure. These data seem to indicate that direct and indirect effects of obesity on cardiovascular disease exist, and that controlling obesity alongside its related complications, most notably diabetes and raised blood pressure, is likely the most effective strategy to reduce the burden of the disease.

Findings from this study are relevant and complement previous evidence from an observational study showing that $46 \%$ (95\% CI 42 to $50 \%$ ) of the excess risk conferred by raised body mass index on coronary artery disease was mediated by effects on blood pressure, glycemia, and cholesterol (proportion mediated was $31 \%$ for blood pressure, $15 \%$ for glycemia, and $10 \%$ for cholesterol) [3]. While the proportion mediated in the previous study for all three intermediate risk factors was significant but slightly lower than in the present study, the use of lifelong cumulative liability to intermediate cardiovascular risk factors might represent and advantage over previous observational studies [4]. However, the lack of a mediation by blood lipids and fasting glucose is paradoxical and might deserve further consideration. For example, emerging data from large-scale randomized clinical trials and genetic epidemiology studies have shown that drugs designed to reduce LDLc or naturally occurred genetic variation at these drug targets appear to increase body weight and impair glycemic control in addition to their cardioprotective effects $[5,6]$. The extent to which 
the use of genetic variants for lipid-drug targets provides evidence of any mediation effects might be of clinical and public health importance. In addition, disglycemia, even in the non-diabetic range and separately from the risk conferred by type 2 diabetes, is a well-stablished cardiovascular risk factors [7], and there is evidence from Mendelian randomization studies showing that poor glycemic control, assessed using $\mathrm{HbA} 1 \mathrm{c}$, appear to mediate much of the effect of obesity on coronary artery disease [8]. While it is possible that nonlinear associations between fasting glucose and cardiovascular risk exists, data are conflicting [9].

The study has several strengths including the use of multivariable Mendelian randomization with inclusion of a vast repertoire of risk factors and cardiovascular outcomes to answer a relevant clinical and public health question. Limitations include the use of data from predominantly European populations, which might limit generalizability to other ethnic groups disproportionately affected by obesity and related metabolic complications. In addition, intermediate cardiometabolic risk factors included in this study are usually present in the context of obesity, therefore the extent to which obesity is even a more powerful mediator of the effect of intermediate risk factors on cardiovascular outcomes would provide additional evidence to target preventive and therapeutic strategies.

With 800 million people worldwide living with obesity, childhood obesity expected to increase by $60 \%$ over the next decade, and medical costs associated with obesity expected to exceed US $\$ 1$ trillion by 2025 , it is clear that current efforts to prevent and treat obesity are inadequate [10]. Evidence from the current study may foster new lines of investigation to gain insights, not only into the underlying mechanisms responsible for the cardiometabolic effect of obesity, but into the etiology of cardiovascular disease itself. Such knowledge have the potential to inform public health and individual strategies to leverage more personalized and efficient approaches to manage cardiovascular risk.

Funding JM was partially supported by the National Institutes of Health (DK40561).

\section{Compliance with ethical standards}

Conflict of interest The author declares no competing interests.

Publisher's note Springer Nature remains neutral with regard to jurisdictional claims in published maps and institutional affiliations.

\section{References}

1. Gill D, Zuber V, Dawson J, Pearson-Stuttard J, Carter AR, Sanderson E, et al. Risk factors mediating the effect of body-mass index and waist-to-hip ratio on cardiovascular outcomes: Mendelian randomization analysis. Int J Obes. 2021. In press

2. Burgess S, Thompson SG. Multivariable Mendelian randomization: the use of pleiotropic genetic variants to estimate causal effects. Am J of Epidemiol. 2015;181:251-60.

3. The Global Burden of Metabolic Risk Factors for Chronic Diseases Collaboration. Metabolic mediators of the effects of bodymass index, overweight, and obesity on coronary heart disease and stroke: a pooled analysis of 97 prospective cohorts with 1.8 million participants. Lancet. 2014;383:970-83.

4. Sanderson E. Multivariable Mendelian randomization and mediation. Cold Spring Harb Perspect Med. 2021;11:a038984.

5. Swerdlow DI, Preiss D, Kuchenbaecker KB, Holmes MV, Engmann JEL, Shah T, et al. HMG-coenzyme A reductase inhibition, type 2 diabetes, and bodyweight: evidence from genetic analysis and randomized rials. Lancet. 2015;385:351-61.

6. Lotta LA, Sharp SJ, Burgess S, Perry JRB, Stewart ID, Willems $\mathrm{SM}$, et al. Association between low-density lipoprotein cholesterol-lowering genetic variants and risk of type 2 diabetes: a meta-analysis. JAMA. 2016;316:1383-91.

7. Merino J, Leong A, Posner DC, Porneala B, Masana L, Dupuis J, et al. Genetically driven hyperglycemia increases risk of coronary artery disease separately from type 2 diabetes. Diabetes Care. 2017;40:687-93.

8. Xu L, Borges MC, Hermani G, Lawlor DA. The role of glycaemic and lipid risk factors in mediating the effect of BMI on coronary heart disease: a two-step, two-sample Mendelian randomisation study. Diabetologia. 2017;60:2210-20.

9. Coutinho M, Gerstein HC, Wang Y, Yusuf S. The relationship between glucose and incident cardiovascular events. A metaregression analysis of published data from 20 studies of 95,783 individuals followed for 12.4 years. Diabetes Care. 1999;22: 233-40.

10. Blüher M. Obesity: global epidemiology and pathogenesis. Nat Rev Endocrinol. 2019;15:288-98. https://doi.org/10.1038/ s41574-019-0176-8. 